\title{
What Factors Are Associated With Femoral Component Internal Rotation in TKA Using the Gap Balancing Technique?
}

\author{
Seung-Yup Lee MD, MSc, Hong-Chul Lim MD, PhD, Ki-Mo Jang MD, PhD, \\ Ji-Hoon Bae MD, PhD (1)
}

Received: 16 November 2016/Accepted: 9 March 2017/Published online: 23 March 2017

(C) The Association of Bone and Joint Surgeons (B) 2017

\begin{abstract}
Background When using the gap-balancing technique for TKA, excessive medial release and varus proximal tibial resection can be associated with internal rotation of the femoral component. Previous studies have evaluated the causes of femoral component rotational alignment with a separate factor analysis using unadjusted statistical methods, which might result in treatment effects being attributed to confounding variables.
\end{abstract}

Each author certifies that neither he, nor any member of his immediate family, have funding or commercial associations (consultancies, stock ownership, equity interest, patent/licensing arrangements, etc) that might pose a conflict of interest in connection with the submitted article.

All ICMJE Conflict of Interest Forms for authors and Clinical Orthopaedics and Related Research ${ }^{\mathbb{R}}$ editors and board members are on file with the publication and can be viewed on request.

Clinical Orthopaedics and Related Research ${ }^{\mathbb{R}}$ neither advocates nor endorses the use of any treatment, drug, or device. Readers are encouraged to always seek additional information, including FDAapproval status, of any drug or device prior to clinical use.

Each author certifies that his institution approved the human protocol for this investigation, that all investigations were conducted in conformity with ethical principles of research, and that informed consent for participation in the study was obtained.

This work was performed at the Korea University Guro Hospital, Seoul, South Korea.

Electronic supplementary material The online version of this article (doi:10.1007/s11999-017-5319-4) contains supplementary material, which is available to authorized users.

S.-Y. Lee, H.-C. Lim

Department of Orthopaedic Surgery, Seoul Barunsesang

Hospital, Seoul, Republic of Korea

K.-M. Jang

Department of Orthopaedic Surgery, Korea University Medical

College, Anam Hospital, Seoul, Republic of Korea
Questions/purposes (1) What pre- and intraoperative factors are associated with internal rotation of the femoral component in TKA using the gap balancing technique? (2) To what degree does femoral component rotation as defined by the navigation system differ from rotation as measured by postoperative CT?

Methods Three hundred seventy-seven knees that underwent computer-assisted primary TKA attributable to degenerative osteoarthritis with varus or mild valgus alignment in which medial soft tissue release was performed, and those with preoperative radiographs including preoperative CT between October 2007 and June 2014 were included in the study. To achieve a balanced mediolateral gap, the structures released during each medial release step were as follows: Step 1, deep medial collateral ligament (MCL); Step 2, superficial MCL (proximal, above the pes anserine tendon) and semimembranosus tendon; and Step 3, the superficial MCL (distal, below the pes anserine tendon). Knees with internal rotation of the femoral component, which was directed by navigation, to achieve a rectangular mediolateral flexion gap were considered cases, and knees without internally rotated femoral components were considered controls. Univariable analysis of the variables (age, sex, BMI, operated side, preoperative hip-knee-ankle angle, preoperative medial proximal tibial angle, preoperative rotation degree of the clinical transepicondylar axis [TEA] relative to the posterior condylar axis [PCA], coronal angle of resected tibia,

\footnotetext{
J.-H. Bae $(\bowtie)$

Department of Orthopaedic Surgery, Korea University Medical

College, Guro Hospital, 148 Gurodong-ro, Guro-gu, Seoul

08308, Republic of Korea

e-mail: osman@kumc.ac.kr; osman@korea.ac.kr
} 
resection of the posterior cruciate ligament, type of prosthesis, and extent of medial release) of cases and controls was performed, followed by a multivariable logistic regression analysis on those factors where $p$ equals 0.15 or less. For an evaluation of navigation error, 88 knees that underwent postoperative CT were analyzed. Postoperative CT scans were obtained for patients with unexplained pain or stiffness after the operations. Using the paired t-test and Pearson's correlation analysis, the postoperative TEAPCA measured with postoperative CT was compared with theoretical TEA-PCA, which was calculated with preoperative TEA-PCA and actual femoral component rotation checked by the navigation system.

Results After controlling for a relevant confounding variable such as postoperative hip-knee-ankle angle, we found that the extent of medial release (Step 1 as reference; Step 2: odds ratio [OR], 5.7, [95\% CI, 2.2-15]; Step 3: OR, 22, [95\% CI, 7.8-62], p < 0.001) was the only factor we identified that was associated with internal rotation of the femoral component. With the numbers available, we found no difference between the mean theoretical postoperative TEA-PCA and the postoperative TEA-PCA measured using postoperative $\mathrm{CT}\left(4.8^{\circ} \pm 2.7^{\circ}\right.$ versus $5.0^{\circ} \pm 2.3^{\circ}$; mean difference, $0.2^{\circ} \pm 1.5^{\circ} ; \mathrm{p}=0.160$ ).

Conclusions Extent of medial release was the only factor we identified that was associated with internal rotation of the femoral component in gap-balancing TKA. To avoid internal rotation of the femoral component, we recommend a carefully subdivided medial-releasing technique, especially for the superficial MCL because once the superficial MCL has been completely released it cannot easily be restored.

Level of Evidence Level III, therapeutic study.

\section{Introduction}

To achieve normal kinetic function of the knee, balanced flexion gap, and appropriate patellar tracking, rotation of the femoral component during TKA has been emphasized [30, 36-39]. There are two methods to determine femoral component rotation in the axial plane: the measured resection technique using the bony landmark and the gap balancing technique $[8,10,11,16]$. The transepicondylar axis (TEA), AP axis, and posterior condylar axis (PCA), which are references used during the measured resection technique, are widely accepted and used [16, 19]. Advocates of the gap balancing technique are concerned with low reproducibility and reliability of the bony landmarks for the measured resection [11, 20, 22].

However, a surgeon using the gap balancing techniquebased TKA occasionally might encounter excessive internal rotation of the femoral component, which can cause postoperative patellofemoral complications, and should be avoided [14, 36]. With the gap balancing technique, excessive medial release and varus proximal tibial resection can be associated with internal rotation of the femoral component (Fig. 1) [6, 8, 11, 14, 18, 25, 38]. Although a rectangular flexion gap can be achieved in these circumstances, resultant kinematic alignment of the distal femur may be abnormal, especially in the patellofemoral joint. Previous studies have evaluated the factors associated with femoral component rotational alignment with a separate factor analysis using unadjusted statistical methods $[6,11,14,25]$. However, without controlling for relevant confounding variables such as extent of medial release, varus proximal tibial resection, and mechanical varus alignment of the knee, it is difficult to identify the strengths of the associations between the factors of interest and femoral component rotation, or whether some of the observed associations were the result of confounding variables.

In the current study we asked: (1) What pre- and intraoperative factors are independently associated with internal rotation of the femoral component in TKA using the gap balancing technique? (2) To what degree does femoral component rotation as defined by the navigation system differ from rotation as measured by a postoperative CT?

\section{Patients and Methods}

Between October 2007 and June 2014, we performed 712 primary TKAs. During that time, 655 knees underwent primary TKA with navigation (92.0\%) (Fig. 2). In all cases, primary TKAs were performed with navigation, except when the navigation system was malfunctioning or unavailable. Inclusion criteria were as follows: the knees with symptomatic degenerative osteoarthritis receiving primary TKA using gap balancing technique-based navigation; varus or mild valgus aligned knees that require medial soft tissue release; and preoperative radiographs including CT. Preoperative CT was performed to identify the original rotational alignment of the femur and tibia. For 61 knees, preoperative CT was declined by the patients, therefore CT was not performed in those patients' knees. In 10 knees, preoperative CT was insufficient for evaluation and these were excluded from the study (Fig. 2). All procedures were performed by the senior author (H-CL). The patients' medical records and radiographs of their knees were reviewed retrospectively. Patients without data regarding computer-assisted surgery, patients who underwent revision TKA, those with inadequate preoperative evaluation (radiography and CT), and those with valgus knees not requiring medial release were excluded. Primary 


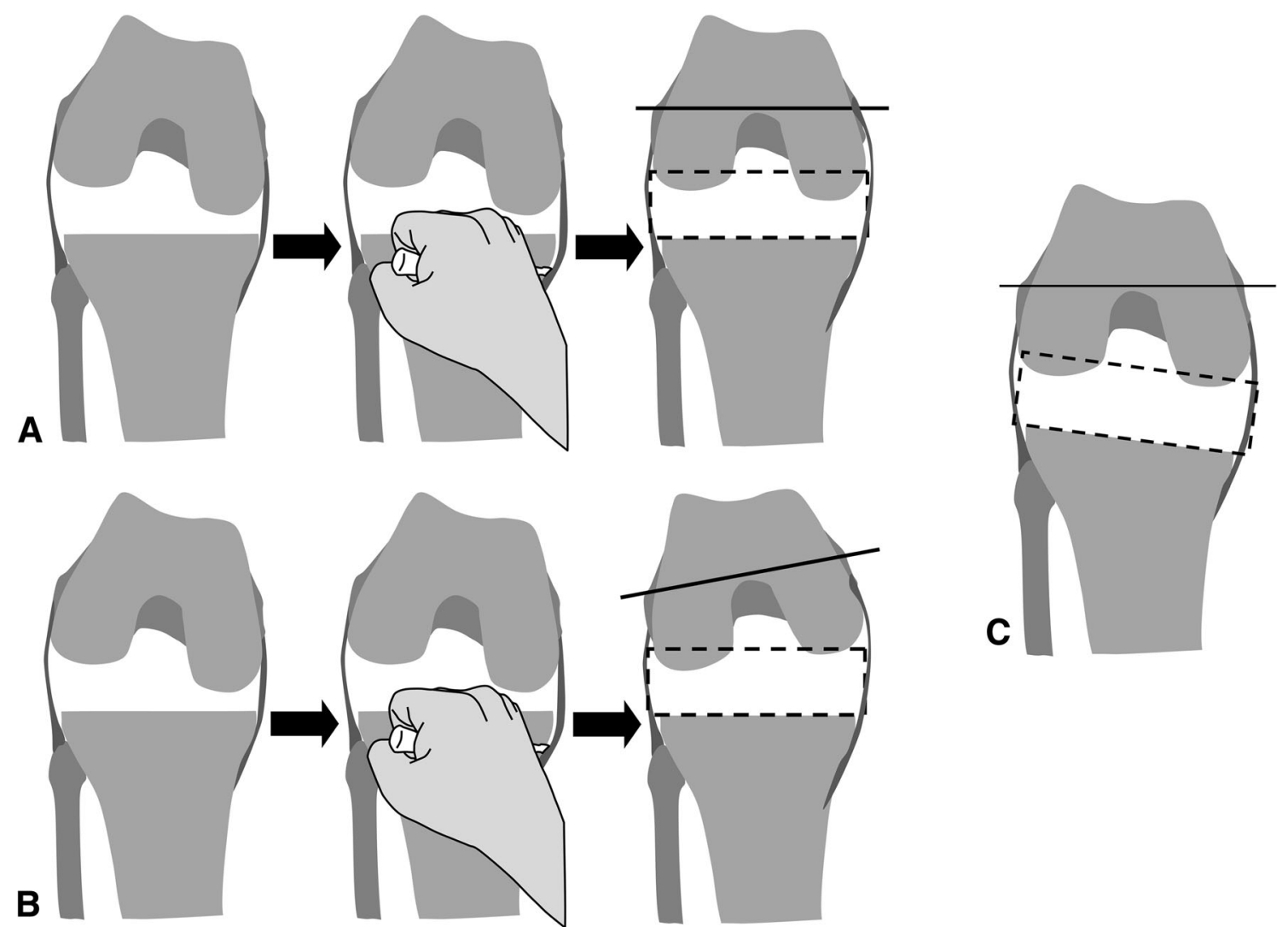

Fig. 1A-C The possible causes of an internally rotated femoral component are shown. (A) In the varus knee, some medial release frequently is required. Adequate medial release results in a rectangular mediolateral flexion gap, which is parallel to the

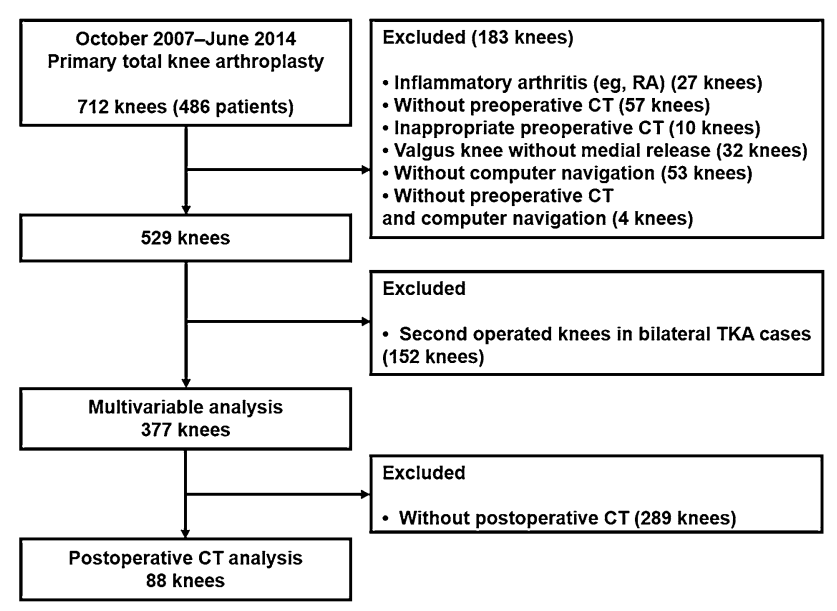

Fig. 2 The study flow diagram is shown. RA = rheumatoid arthritis.

TKAs for inflammatory arthritis such as rheumatoid arthritis also were excluded (Fig. 2). In cases of bilateral TKAs, only the first surgically treated knee was included. After application of these criteria, 377 knees were included transepicondylar axis. (B) An overly performed medial release will result in an internally rotated femoral component relative to the transepicondylar axis. (C) Inadvertent varus resection of the proximal tibia will cause internal rotation of the femoral component.

for our first research question regarding univariable and multivariable analysis of femoral component internal rotation (Table 1).

According to femoral component rotation directed by the navigation system to achieve a rectangular flexion gap, the entire cohort was divided in two groups: one group with an internally rotated femoral component (Group IR, cases) and one group with a neutrally or an externally rotated femoral component (Group non-IR, controls). To analyze what degree femoral component rotation, as defined by the navigation system, differs from rotation as measured by postoperative CT, a total of 88 knees were included. Postoperative CTs were performed in 88 patients (131 knees) with unexplained pain or stiffness after surgery to identify malrotation of the components. In bilateral TKAs, the first 43 knees that underwent TKA were selected. The 88 knees included seemed representative of the larger population in that the variables we analyzed (Table 1) were not different in those of the entire population ( 377 knees) (Appendix 1. Supplemental material is available with the online version of $\left.C O R R^{\circledR}\right)$. 
Table 1. Case summary $(\mathrm{n}=377)$

\begin{tabular}{|c|c|}
\hline Variables & Mean \pm SD (range) or frequency \\
\hline Age (years) & $69 \pm 6.3(50-90)$ \\
\hline Sex (male:female) & $43: 334$ \\
\hline BMI $\left(\mathrm{kg} / \mathrm{m}^{2}\right)$ & $25 \pm 2.1(20.7-31)$ \\
\hline Surgically treated side (right:left) & $185: 192$ \\
\hline Preoperative hip-knee-ankle angle $\left({ }^{\circ}\right.$; positive value, varus $)$ & $9.7 \pm 5.8(-4.8$ to 29$)$ \\
\hline Preoperative medial proximal tibial angle $\left({ }^{\circ}\right)$ & $84.4 \pm 3.5(71-96)$ \\
\hline $\begin{array}{l}\text { Preoperative transepicondylar axis-posterior condylar axis }{ }^{*} \\
\quad\left({ }^{\circ} \text {; positive value, external rotation) }\right.\end{array}$ & $5.8 \pm 2.5(0-13)$ \\
\hline PCL resection (yes:no) & $146: 231$ \\
\hline $\begin{array}{l}\text { Type of prosthesis (e. motion }{ }^{\circledR} \text { : Columbus }{ }^{\circledR} \text { : e. motion }{ }^{\circledR} \\
\text { Pro; all from B. Braun Aesculap }{ }^{\circledR} \text {, Tuttlingen, Germany) }\end{array}$ & 233:81:63 \\
\hline Coronal angle of the resected tibia ${ }^{\dagger}\left({ }^{\circ}\right.$; positive value, varus resection of the tibia) & $-0.0 \pm 0.8(-2$ to 2$)$ \\
\hline Extent of the medial release (Step 1:Step 2:Step 3) & 218:119:40 \\
\hline $\begin{array}{l}\text { Femoral component rotation recommended to obtain the rectangular } \\
\text { flexion gap during femoral planning } \operatorname{step}^{\dagger}\left({ }^{\circ} ; \text { positive value, external rotation }\right)\end{array}$ & $1.1 \pm 1.9(-5$ to 7$)$ \\
\hline Femoral component rotation measured by the AP femoral cutting $\mathrm{jig}^{\dagger}\left({ }^{\circ}\right.$; positive value, external rotation) & $1.1 \pm 1.9(-4$ to 8$)$ \\
\hline Postoperative hip-knee-ankle angle $\left({ }^{\circ}\right.$; positive value, varus $)$ & $1.5 \pm 2.4(-5.1$ to 9.0$)$ \\
\hline
\end{tabular}

*Using CT, the rotational angle of the clinical epicondylar axis relative to the posterior condylar axis was measured; ${ }^{\dagger}$ values were acquired from navigation data.

The current study was approved by the ethics committee of Korea University Guro Hospital (institutional review board approval number: KUGH14148).

The standard medial parapatellar approach was used. After exposing the knee, medial osteophytes were removed and tibial insertions of the deep medial collateral ligament (MCL) and posterior oblique ligament were released along the medial meniscus, 3 to $5 \mathrm{~mm}$ below the medial joint line. Further steps of medial release, if performed, are described later. A computer navigation system (OrthoPilot $^{\mathbb{R}}$ navigation system; B. Braun Aesculap ${ }^{\mathbb{R}}$, Tuttlingen, Germany) was used. Gap balancing-based computer navigation was used for primary TKA. Resection of the tibia was performed before femoral resection. Detailed surgical steps and manipulation of the navigation system were performed as recommended by the manufacturer. The prostheses implanted, e.motion ${ }^{\mathbb{R}}(233$ knees $)$, Columbus ${ }^{\circledR}$ (81 knees), and e.motion ${ }^{\circledR}$ Pro (63 knees) (B. Braun Aesculap ${ }^{\mathbb{R}}$ ), varied according to time when the TKA was performed. The first implant was used for the first 47 months of the study, and the second implant was used for the next 19 months. The last implant was used for the final 14 months of the study. The types of implants were determined according to the condition of the PCL and the feasibility of a balanced extension-flexion gap. For example, we used a PCL-substituting implant when the PCL showed severe degeneration or laxity, or when the flexion gap was too tight compared with the extension gap. A cruciate retaining type was used in 231 knees (61\%), and posterior substituting type was used in 146 knees (39\%).

After registration of the bony landmarks, the proximal tibial resection was performed (Fig. 3). The varus-valgus angle of the resected tibia was checked (Fig. 3A). After proximal tibial resection, extension and flexion gaps were measured using a gap tensioning device. If it was decided that the PCL should be resected because of its consistency and the chosen implant, then resection was performed before gap measurement. With these gap data, the femoral resection planning step was performed (Fig. 3B). To obtain a rectangular space for the extension and flexion gaps, greater than $2 \mathrm{~mm}$ mediolateral asymmetry was not permitted according to our navigation data. When further medial release (mediolateral asymmetry $>2 \mathrm{~mm}$ ) was needed according to the navigation gap data, stepwise medial release was performed as follows: Step 1, removal of medial osteophytes and release of tibial insertions of the deep MCL and posterior oblique ligament (this step was performed before the initial femoral planning step); Step 2, partial release of the tibial insertion of the superficial MCL above the level of the pes anserine tendon and direct arm of the semimembranosus tendon; and Step 3, near complete release of the tibial insertion of the superficial MCL below the level of the pes anserine tendon (Fig. 4) $[3,23,24,31,39]$. In the case of multiple medial release steps, gap checking and femoral planning were repeated before the femoral AP resection. 
Fig. 3A-C Important navigation procedures are shown. (A) After registration of bony landmarks and hip, knee, and ankle centers, the proximal tibial resection was performed. Varus-valgus angle of the resected tibia was checked (asterisk). (B) In the femoral planning step, to achieve a rectangular mediolateral gap in knee extension and flexion, varus-valgus angle and rotation (dagger) of the femoral component are adjusted with navigation dictation. The numbers displayed next to femurs and tibias represent expected amounts of bone resection. (C) After distal femoral resection, the rotational position of the AP femoral cutting jig on the distal femur (double dagger), which is positioned manually, is displayed in real time. This represents actual femoral component rotation. In this case, the femoral component is in $2^{\circ}$ external rotation relative to the posterior condylar axis. Green $=$ this value is as planned; Ext. = extension.
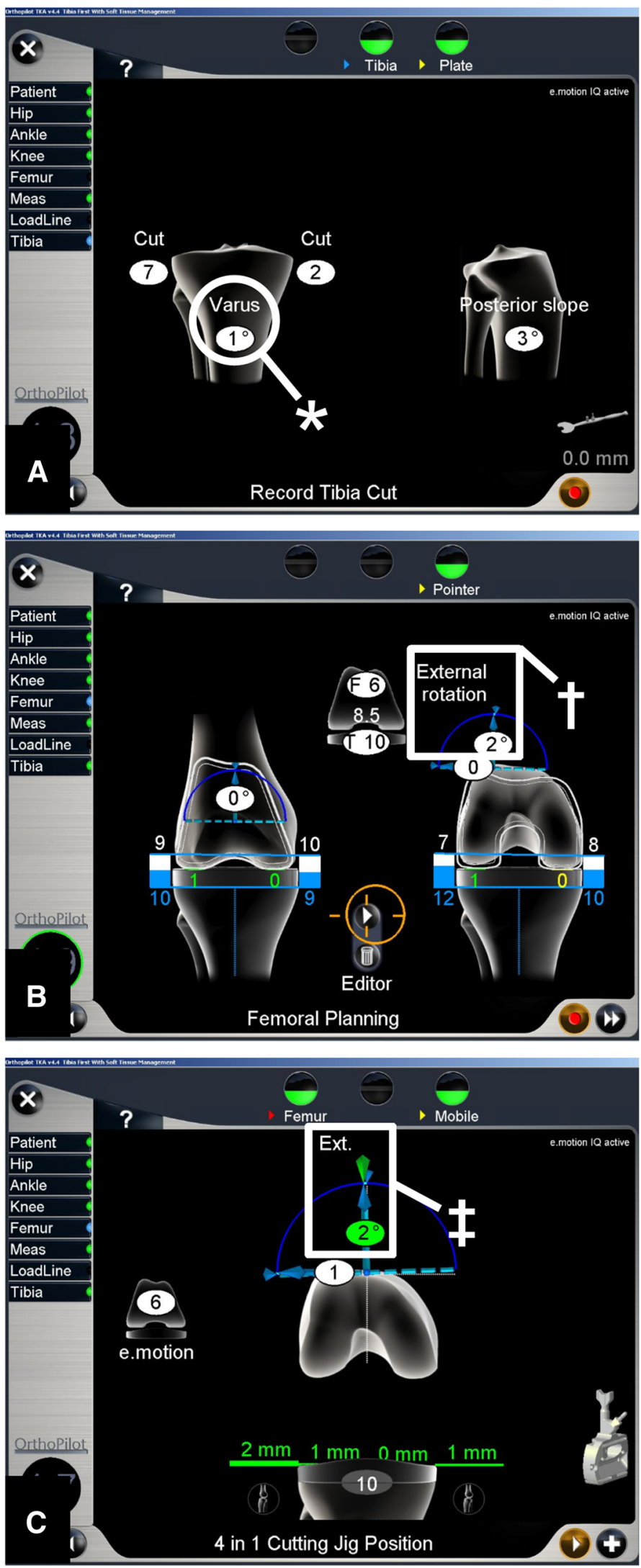


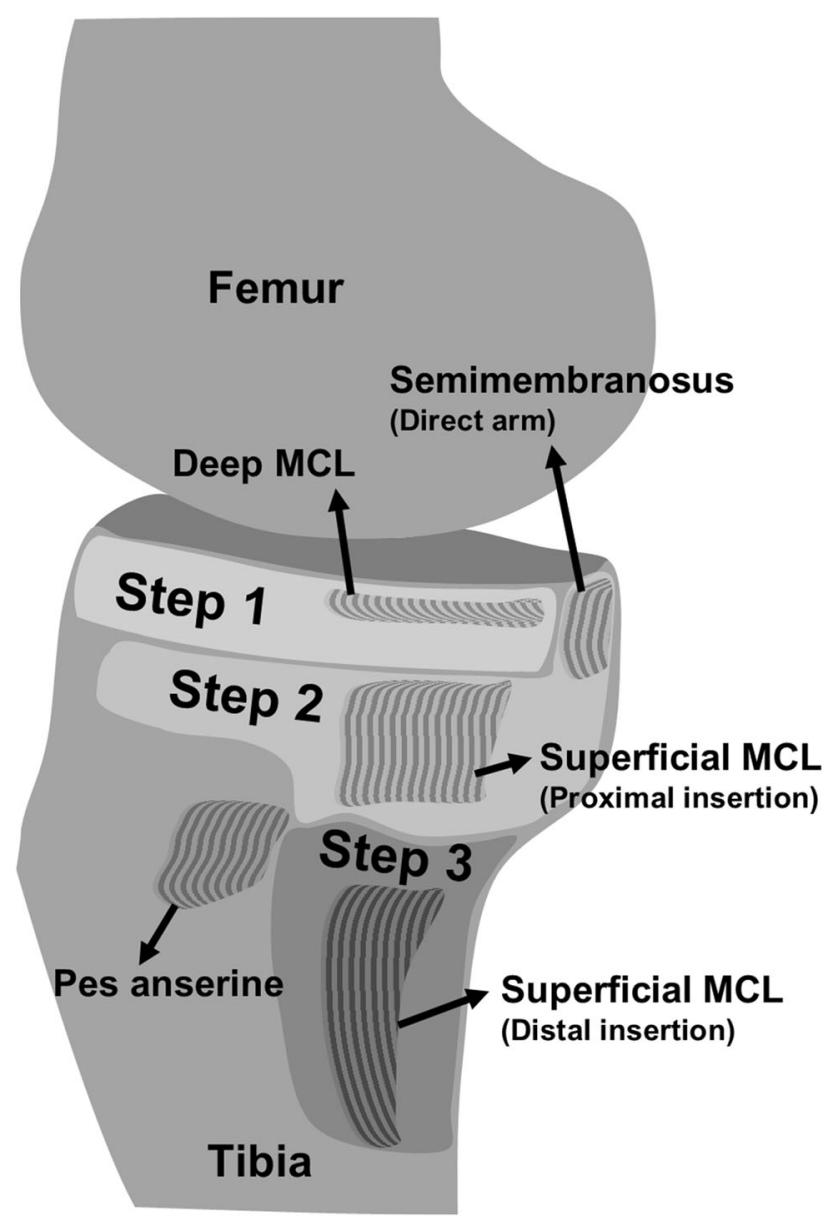

Fig. 4 The medial release steps are shown. Removal of medial osteophytes and release of tibial insertions of the deep MCL and posterior oblique ligament are performed in Step 1. If further medial release was required, the proximal insertion of the superficial MCL (above the pes anserine tendon) and anterior arm of the semimembranosus were released (Step 2). In the final step, Step 3, the distal insertion of the superficial MCL (below pes anserine tendon) was released. $\mathrm{MCL}=$ medial collateral ligament.

In addition to medial and lateral gaps of extension and flexion, femoral component rotation directed by the computer was recorded when the symmetric mediolateral space was achieved (Fig. 3B). The navigation system used in the current study measures rotation of the femoral component based on the PCA. According to the planned femoral component rotation, the entire cohort was divided into cases and controls. As planned in this step, distal, anterior, and posterior femoral resections were performed. However, there can be a slight difference between the planned femoral component rotation and actual rotational position of the femoral cutting jig owing to possible error during manual placement of the jig. Therefore, jig rotation measured just before AP resection of the femur was recorded (actual femoral component rotation) (Fig. 3C).

Using standing AP and lateral radiographs, pre- and postoperative hip-knee-ankle angle and preoperative medial proximal tibial angle were measured. A positive pes anserine tendon value indicates a varus knee. Using the preoperative $\mathrm{CT}$ of the knee, the amount of rotation of the clinical TEA relative to the PCA (TEA-PCA) was measured (Fig. 5A) [38]. A positive TEA-PCA value indicates external rotation of the TEA relative to the PCA. Postoperative CT was performed in 88 knees (Fig. 5B). All postoperative radiologic evaluations were performed 1 to 2 weeks after surgery. In these knees, postoperative TEAPCA, which was measured using CT, was compared with the theoretical postoperative TEA-PCA. Theoretical postoperative TEA-PCA was calculated as: preoperative TEAPCA minus actual femoral component rotation (Fig. 5C). For example, with $5^{\circ}$ external rotation for preoperative TEA-PCA and $2^{\circ}$ external rotation for actual femoral component rotation, the theoretical postoperative TEAPCA is $3^{\circ}$ of external rotation. If the theoretical postoperative TEA-PCA is significantly different from the postoperative TEA-PCA, which was measured using postoperative $\mathrm{CT}$, then this indicates an error during the navigation procedure. Compared with postoperative TEAPCA, theoretical postoperative TEA-PCA not within a tolerance level of $\pm 3^{\circ}$ was considered an outlier [13, 26, 29, 41]. All radiologic measurements were performed twice during a 2-week period by two orthopaedic surgeons (SYL and KMJ).

\section{Statistical Analysis}

The reliability of radiologic measurements was evaluated using intraclass correlation coefficients (ICCs) [1]. Comparisons of gaps and their changes according to each compartment and extension-flexion were performed using one-way ANOVA testing followed by Tukey's post hoc test. The differences in these analyses were considered significant at a probability of 0.05 or less.

The variables of Group IR and Group non-IR were compared using an independent t-test, Fisher's exact test, and linear-by-linear association analysis. Cases were defined as having internal rotation of the planned femoral component rotation. Univariable analysis of the variables (age, sex, BMI, surgically treated side, preoperative hipknee-ankle angle, preoperative medial proximal tibial angle, preoperative TEA-PCA, PCL resection, type of prosthesis, coronal angle of the resected tibia, and extent of medial release) of cases (Group IR) and controls (Group non-IR) was performed. Only covariates with a resulting $p$ value of 0.15 or less were included in the backward stepwise multivariable logistic regression analysis. The number of cases (Group IR) in the current study was 39 (prevalence, $10.3 \%$ ); two variables were included in the multivariable logistic regression analysis. The required 
Fig. 5A-C Comparisons between the theoretical postoperative TEA-PCA and postoperative TEA-PCA measured using postoperative CT are shown. (A) Preoperative TEA-PCA is measured using preoperative $\mathrm{CT}$, and (B) postoperative TEA-PCA is measured using postoperative CT. (C) Theoretical postoperative TEA-PCA is calculated by subtracting the preoperative TEA-PCA from the rotational position of the AP femoral cutting jig on the distal femur (actual femoral component rotation [F-Rot]; Fig. 3C). For example, with $5^{\circ}$ external rotation for preoperative TEA-PCA and $2^{\circ}$ external rotation for actual femoral component rotation, the theoretical postoperative TEA-PCA is $3^{\circ}$ external rotation. If the theoretical postoperative TEA-PCA is significantly different using the postoperative TEA-PCA, which was measured using postoperative $\mathrm{CT}$, then this indicates an error during the navigation procedure. TEA $=$ transepicondylar axis; $\mathrm{PCA}=$ posterior condylar axis; Ext. $=$ extension.
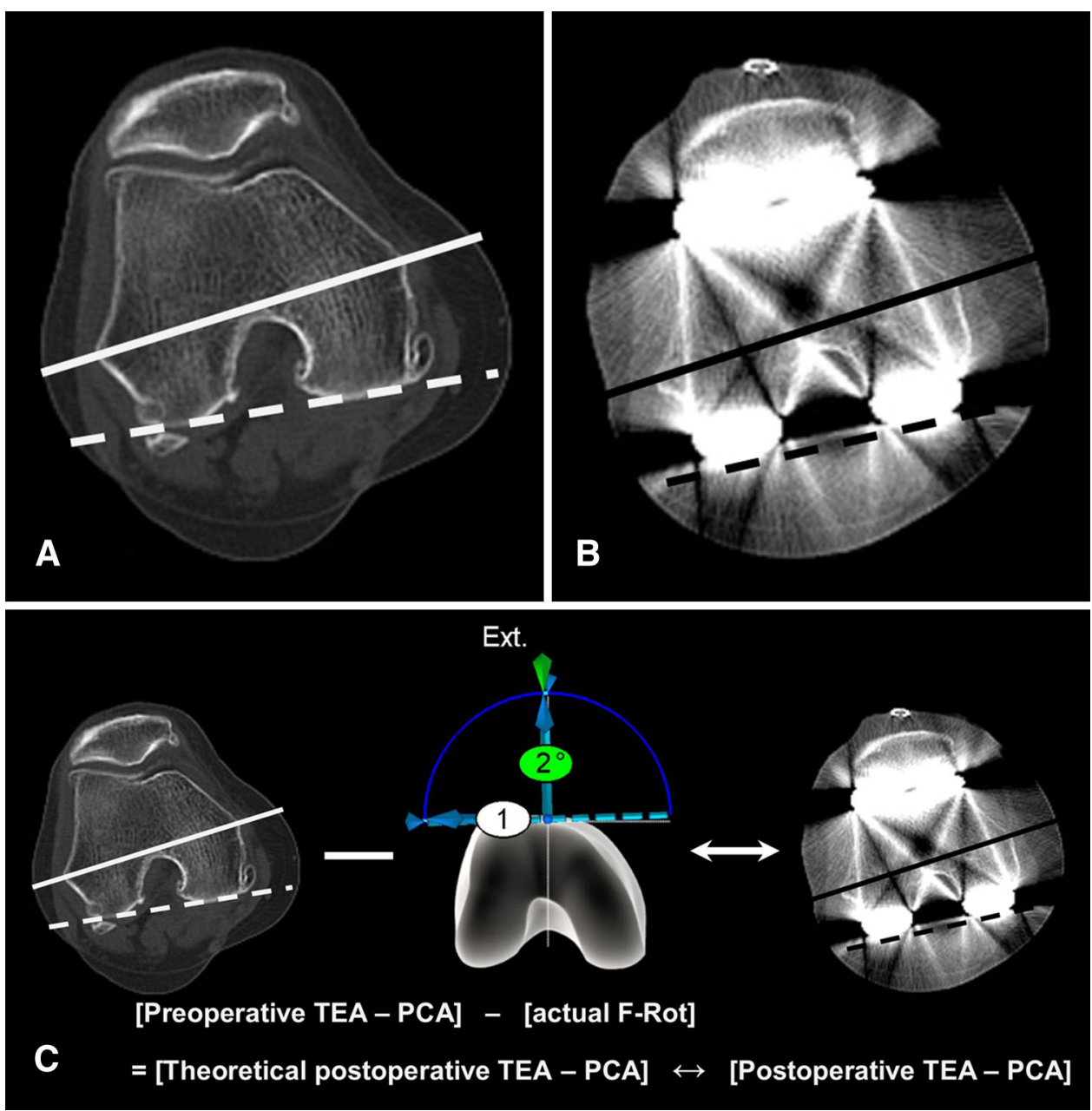

sample size was approximately $194(2 \times 10 / 0.103)$ [35]. Regarding multicollinearity between the variables included in the multivariable analysis, a variance inflation factor greater than 5 was considered indicative of collinearity [15]. To analyze the navigation error in the axial plane, theoretical postoperative TEA-PCA and postoperative TEA-PCA measured using postoperative CT were compared using the paired t-test and Pearson's correlation analysis. A probability of 0.05 or less indicated significance. Statistical analyses were performed using SPSS, Version 20.0 (IBM Corp, Armonk, NY, USA).

All inter- and intraobserver ICCs showed excellent agreement regarding radiographic measurement reliability (Table 2). As progressively larger releases were performed, we found the medial gaps in the flexed knee increased more than the medial gap in the extended knee, which was greater than the lateral gaps in the flexed or extended knee (Table 3). In the demographic comparison between two groups, the mean planned femoral component rotation of Group IR and Group non-IR were $-2.0^{\circ} \pm 1.1^{\circ}$ and 1 . $4^{\circ} \pm 1.6^{\circ}$, respectively. The preoperative hip-knee-ankle angle of Group IR was greater than that of Group non-IR.
The proportions of the larger medial release steps (Steps 2 and 3) were higher in Group IR than Group non-IR (Table 4). Univariable factor analysis showed that the preoperative hip-knee-ankle angle (odds ratio [OR], 1.1; 95\% CI, 1.0-1.1; $\mathrm{p}=0.037$ ) and extent of medial release (Step 1 as reference; Step 2: OR, 5.9, [95\% CI, 2.3-15]; Step 3: OR, 23, [95\% CI, 8.4-66], p < 0.001) were selected for multivariable analysis $(\mathrm{p} \leq 0.15)$ (Table 5$)$.

\section{Results}

After controlling for relevant confounding variables such as the preoperative hip-knee-ankle angle, preoperative TEA-PCA, and coronal angle of the resected tibia, we found that the extent of medial release (Step 1 as reference; Step 2: OR, 5.7, [95\% CI, 2.2-15); Step 3: OR, 22, [95\% $\mathrm{CI}, 7.8-62], \mathrm{p}<0.001$ ) was the only factor we identified that was associated with internal rotation of the femoral component (Table 6). All variance inflation factors of these covariates showed values less than 5 , which indicates that there is no serious multicollinearity between the covariates. 
Table 2. Intraclass correlation coefficients for radiologic evaluations

\begin{tabular}{llllll}
\hline Observer & $\begin{array}{l}\text { Preoperative hip- } \\
\text { knee-ankle angle }\end{array}$ & $\begin{array}{l}\text { Postoperative hip- } \\
\text { knee-ankle angle }\end{array}$ & $\begin{array}{l}\text { Preoperative medial } \\
\text { proximal tibial angle }\end{array}$ & $\begin{array}{l}\text { Preoperative } \\
\text { TEA-PCA }\end{array}$ & Postoperative TEA-PCA \\
\hline $\begin{array}{l}\text { Interobserver } \\
\text { ICC (95\% CI) }\end{array}$ & $0.984(0.962-0.993)$ & $0.953(0.888-0.980)$ & $0.848(0.641-0.935)$ & $0.807(0.546-0.918)$ & $0.822(0.580-0.924)$ \\
$\begin{array}{l}\text { Observer 1 } \\
\text { ICC (95\% CI) }\end{array}$ & $0.995(0.987-0.998)$ & $0.984(0.963-0.993)$ & $0.925(0.824-0.968)$ & $0.875(0.704-0.947)$ & $0.855(0.658-0.939)$ \\
$\begin{array}{l}\text { Observer 2 } \\
\text { ICC (95\% CI) }\end{array}$ & $0.993(0.983-0.997)$ & $0.903(0.772-0.959)$ & $0.820(0.575-0.923)$ & $0.837(0.617-0.931)$ & $0.813(0.558-0.920)$ \\
\hline
\end{tabular}

ICC $=$ intraclass correlation coefficient; TEA = transepicondylar axis; PCA = posterior condylar axis.

Table 3. Comparisons of gaps and their changes according to each compartment and extension-flexion $(\mathrm{n}=377)$

\begin{tabular}{|c|c|c|c|c|}
\hline \multirow{2}{*}{$\begin{array}{l}\text { Step } \\
\text { Release step }(\mathrm{mm}, \text { mean } \pm \mathrm{SD})\end{array}$} & \multicolumn{2}{|c|}{ Knee extension } & \multicolumn{2}{|c|}{ Knee flexion } \\
\hline & Medial & Lateral & Medial & Lateral \\
\hline 1 & $9.0 \pm 2.5$ & $11 \pm 2.3$ & $9.2 \pm 2.7$ & $11.4 \pm 2.8$ \\
\hline 2 & $9.6 \pm 2.3$ & $11 \pm 2.1$ & $10 \pm 2.8$ & $11.9 \pm 2.4$ \\
\hline 3 & $12 \pm 2.0$ & $12 \pm 2.2$ & $12 \pm 2.7$ & $11.9 \pm 2.2$ \\
\hline p Value ${ }^{*}$ & $<0.001$ & 0.217 & $<0.001$ & 0.095 \\
\hline \multicolumn{5}{|l|}{ Post hoc analysis (p value ${ }^{\dagger}$ ) } \\
\hline Step 1 vs 2 & 0.045 & 0.786 & $<0.001$ & 0.109 \\
\hline Step 2 vs 3 & 0.016 & 0.421 & 0.006 & 1.000 \\
\hline Step 1 vs 3 & $<0.001$ & 0.202 & $<0.001$ & 0.493 \\
\hline \multicolumn{5}{|l|}{ Gap change $(\mathrm{mm}$, mean $\pm \mathrm{SD})$} \\
\hline Steps 1 and $2^{*}$ & $1.6 \pm 1.5$ & $0.6 \pm 1.2$ & $2.3 \pm 1.9$ & $0.9 \pm 1.5$ \\
\hline Steps 2 and $3^{\S}$ & $1.6 \pm 1.4$ & $0.2 \pm 0.8$ & $2.8 \pm 2.1$ & $0.4 \pm 1.2$ \\
\hline
\end{tabular}

*Compartment gaps according to release steps were analyzed with the one-way ANOVA; ${ }^{\dagger}$ Tukey's post hoc tests were performed to compare two different medial release steps; "between Steps 1 and 2, gap changes in the four compartments showed significant differences (p $<0.001$; oneway ANOVA); ${ }^{\S}$ between Steps 2 and 3, gap changes in the four compartments showed significant differences (p <0.001; one-way ANOVA). Tukey's post hoc test showed a significant difference between the two different compartments, except between the lateral compartment in knee extension and in knee flexion.

With the numbers available, we found no difference between the mean theoretical postoperative TEA-PCA and the postoperative TEA-PCA measured using postoperative $\mathrm{CT}\left(4.8^{\circ} \pm 2.7^{\circ}\right.$ versus $5.0^{\circ} \pm 2.3^{\circ}$; mean difference, $0.2^{\circ}$ $\pm 1.5^{\circ} ; \mathrm{p}=0.160$ ). There was a strong positive correlation between the theoretical and postoperative values of TEAPCA $(r=0.838 ; p<0.001)$, meaning that as theoretical TEA-PCA increased, so did postoperative TEA-PCA. Among the 88 knees, only three $(3.4 \%)$ showed a difference exceeding $\pm 3^{\circ}$ for the theoretical and actual postoperative TEA-PCA values.

\section{Discussion}

Because excessive internal rotation of the femoral component can be associated with patellofemoral tilt, subluxation, and dislocation, it is important to achieve correct rotational alignment of the femoral component $[14,36]$. Although studies identified several factors, such as excessive medial release and varus proximal tibial resection, that were associated with femoral component internal rotation during gap balancing technique-based TKA, the studies evaluated the factors using unadjusted statistical methods $[6,11,14,25]$. Because this approach does not take into account the influence of possible confounding variables, simple comparisons without adjustment of this sort are potentially misleading. To overcome this problem, we performed multivariable analysis, controlling for relevant confounding variables such as extent of medial release, varus proximal tibial resection, and mechanical varus alignment of the knee. The mostimportant finding of the current study is that the extent of medial release is the only factor we identified that was associated with internal rotation of the femoral component in TKAs performed using the gap balancing technique. We 
Table 4. Comparison according to femoral component rotation directed by the navigation system to obtain a rectangular mediolateral flexion gap

\begin{tabular}{|c|c|c|c|}
\hline Variables & $\begin{array}{l}\text { Group non-IR } \\
(\mathrm{n}=338)\end{array}$ & Group IR $(\mathrm{n}=39)$ & $\mathrm{p}$ Value \\
\hline Age $^{*}$ (years) & $69 \pm 6.4$ & $67 \pm 5.9$ & $0.185^{\dagger}$ \\
\hline Sex (male:female) & $39: 299$ & $4: 35$ & $0.812^{*}$ \\
\hline $\operatorname{BMI}^{*}\left(\mathrm{~kg} / \mathrm{m}^{2}\right)$ & $25 \pm 2.1$ & $25 \pm 2.1$ & $0.249^{\dagger}$ \\
\hline Surgically treated side (right:left) & $169: 169$ & $16: 23$ & $0.288^{\ddagger}$ \\
\hline Preoperative kip-knee-ankle angle ${ }^{*}\left({ }^{\circ}\right.$; positive value, varus) & $9.5 \pm 5.7$ & $11.6 \pm 6.0$ & $0.036^{\dagger}$ \\
\hline Preoperative medioproximal tibial angle ${ }^{*}\left({ }^{\circ}\right)$ & $84 \pm 3.5$ & $84 \pm 3.5$ & $0.850^{\dagger}$ \\
\hline Preoperative TEA-PCA ${ }^{*}\left({ }^{\circ}\right.$; positive value, external rotation $)$ & $5.9 \pm 2.5$ & $5.4 \pm 2.5$ & $0.289^{\dagger}$ \\
\hline PCL resection (yes:no) & $132: 206$ & $14: 25$ & $0.702^{\ddagger}$ \\
\hline $\begin{array}{l}\text { Type of prosthesis (e. motion }{ }^{\mathbb{R}}: \text { Columbus }^{\mathbb{R}}: \text { e. } \text { motion }^{\circledR} \text { Pro; all from } \\
\text { B. Braun Aesculap }{ }^{\mathbb{R}} \text {, Tuttlingen, Germany) }\end{array}$ & 205:75:58 & 28:6:5 & $0.231^{\S}$ \\
\hline Coronal angle of the resected tibia ${ }^{*}, \|\left({ }^{\circ}\right.$; positive value, varus resection of the tibia) & $-0.0 \pm 0.8$ & $0.1 \pm 0.7$ & $0.351^{\dagger}$ \\
\hline Extent of the medial release (Step 1:Step 2:Step 3) & 212:102:24 & $6: 17: 16$ & $<0.001^{\S}$ \\
\hline $\begin{array}{l}\text { Femoral component rotation recommended to obtain the rectangular } \\
\text { flexion gap*, } \| \text { (planned femoral component rotation) }\left({ }^{\circ} \text {; positive value, }\right. \\
\text { external rotation) }\end{array}$ & $1.4 \pm 1.6$ & $-2.0 \pm 1.1$ & $<0.001^{\dagger}$ \\
\hline $\begin{array}{l}\text { Femoral component rotation measured by the AP femoral cutting jig }{ }^{*} \text {, } \\
\| \text { (actual femoral component rotation) }\left({ }^{\circ} ; \text { positive value, external rotation) }\right.\end{array}$ & $1.4 \pm 1.7$ & $-1.9 \pm 1.0$ & $<0.001^{\dagger}$ \\
\hline Postoperative hip-knee-ankle angle ${ }^{*}\left({ }^{\circ}\right.$; positive value, varus $)$ & $1.5 \pm 2.4$ & $1.1 \pm 2.3$ & $0.311^{\dagger}$ \\
\hline
\end{tabular}

$\mathrm{IR}=$ internal rotation; TEA = transepicondylar axis; PCA = posterior condylar axis; *values given as the mean $\pm \mathrm{SD} ;{ }^{\dagger} \mathrm{p}$ values were calculated using independent t-tests; ${ }^{\ddagger} \mathrm{p}$ values were calculated using Fisher's exact tests; ${ }^{\S} \mathrm{p}$ values were determined with the linear-by-linear association

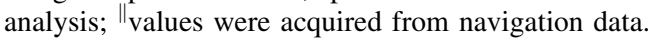

found that a near-complete release of the superficial MCL tibial insertion below the level of the pes anserine tendon was consistently associated with internal rotation of the femoral component. Once complete release of the superficial MCL has been performed, it cannot be undone to overcome internal rotation of the femoral component, therefore a carefully subdivided medial-releasing technique, particularly for the superficial MCL seems important to prevent internal rotation of the femoral component. Calculating with femoral rotation profiles of navigation records, preoperative and postoperative CT, errors during navigation referencing were not suspected.

Our study has several limitations. The first is patient selection. Although we tried to use navigation whenever possible except when the system was not working or available, there may be selection bias owing to the patients who did not receive this technology. For the second question of the current study, only a limited number of patients underwent postoperative CT. Although these patients were not substantially different from the entire population, patients who had postoperative $\mathrm{CT}$ underwent additional imaging because of unexplained pain or stiffness after the arthroplasty. We could not ethically perform postoperative $\mathrm{CT}$ in all patients, therefore we limited it to patients with a notable problem, and an accurate rotation profile was needed. With this limited number of cases, we could not integrate the navigation accuracy to the covariate of multivariable analysis. Second, there could be some overlap among the medial release steps because there is no definite boundary between medial soft tissue structures. However, this possibility seems to be minimal because the medial release technique used in our study was simplified to three steps compared with that used in previous studies $[17,31]$. Although there are numerous medial release techniques that differ in sequence and structures released, Hunt et al. [17] noted that there is a lack of evidence supporting current methods. Furthermore, it is difficult to distinguish each medial fiber structure during surgery, therefore alternative medial release methods other than the traditional medial subperiosteal release technique have been proposed $[2,33]$. In addition, preoperative medial ligamentous laxity or contracture, which can affect medial gaps, was not evaluated. Third, there could be another selection bias in type of implant and in PCL resection. Although both covariates showed little association with femoral component internal rotation in our analysis, the decision regarding PCL resection and implant type were made by the surgeon subjectively, and by time sequence (the implants were used sequentially during a period of years), respectively. Future randomized studies of these covariates may be required to decrease the issue of selection bias. 
Table 5. Univariable analysis* of factors associated with femoral component internal rotation directed by the navigation system to obtain a rectangular mediolateral flexion gap $(\mathrm{n}=377)$

\begin{tabular}{|c|c|c|c|}
\hline Factor & Odds ratio & $95 \% \mathrm{CI}$ & $\mathrm{p}$ Value \\
\hline Age & 1.0 & $0.9-1.0$ & 0.185 \\
\hline Sex & & & 0.812 \\
\hline \multicolumn{4}{|l|}{ Male (reference) } \\
\hline Female & 1.1 & $0.4-3.4$ & \\
\hline BMI & 0.9 & $0.8-1.1$ & 0.249 \\
\hline Surgically treated side & & & 0.290 \\
\hline \multicolumn{4}{|l|}{ Right (reference) } \\
\hline Left & 1.4 & $0.7-2.8$ & \\
\hline Preoperative hip-knee-ankle angle & 1.1 & $1.0-1.1$ & $0.037^{\dagger}$ \\
\hline Preoperative medial proximal tibial angle & 1.0 & $0.9-1.1$ & 0.850 \\
\hline Preoperative TEA-PCA & 0.9 & $0.8-1.1$ & 0.289 \\
\hline PCL resection & & & 0.702 \\
\hline \multicolumn{4}{|l|}{ No (reference) } \\
\hline Yes & 0.9 & $0.44-1.7$ & \\
\hline \multicolumn{4}{|l|}{ e. motion ${ }^{\mathbb{R}}$ (reference) } \\
\hline Columbus ${ }^{\circledR}$ & 0.6 & $0.2-1.5$ & \\
\hline e. motion ${ }^{\mathbb{R}}$ Pro & 0.6 & $0.2-1.7$ & \\
\hline Coronal angle of the resected tibia & 1.2 & $0.8-1.9$ & 0.350 \\
\hline Extent of the medial release & & & $<0.001^{\dagger}$ \\
\hline \multicolumn{4}{|l|}{ Step 1 (reference) } \\
\hline Step 2 & 5.9 & $2.3-15$ & \\
\hline Step 3 & 24 & $8.4-66$ & \\
\hline
\end{tabular}

TEA = transepicondylar axis; PCA = posterior condylar axis; all prostheses from B. Braun Aesculap ${ }^{\mathbb{B}}$, Tuttlingen, Germany; $*$ using univariable analysis, only covariates with resulting $\mathrm{p}$ value less than 0.15 were included in the backward stepwise multivariable logistic regression analysis; ${ }^{\dagger}$ covariates with a $\mathrm{p}$ less than 0.15 were included in multivariable analysis.

Table 6. Multivariable analysis* of factors associated with femoral component internal rotation

\begin{tabular}{llll}
\hline Factor & Odds ratio & $95 \%$ CI & p Value \\
\hline Preoperative hip-knee-ankle angle & 1.0 & $1.0-1.1$ & \\
Extent of the medial release & & & \\
Step 1 (reference) & & & \\
Step 2 & 5.7 & $2.2-5$ & \\
Step 3 & 22 & $7.8-62$ \\
\hline
\end{tabular}

*Using multivariable analysis, covariates with a resulting $\mathrm{p}$ value less than 0.05 were considered significant.

Numerous studies have shown a relationship between medial release and increment of the medial tibiofemoral gap $[5,6,21,27,31,42]$. In the current study, gap increment according to each compartment showed that the medial flexion gap was the most-increased compartment, followed by the medial extension gap, as a progressively larger release was done. This finding was consistent with those of previous studies [5, 28, 43]. Excessive internal or external rotation of the femoral component could happen during the gap balancing technique, which uses the tibial cut surface as a reference for femoral component rotation $[11,14,25,32,34,38]$. Too much release of the medial structures may result in symptomatic instability, use of very thick polyethylene bearings, or even constrained implants to address the excessive medial gap [40]. Thicker polyethylene bearings and constrained implants are associated with a higher risk of loosening and joint-line elevation $[4,39,40]$. In addition, uneven load distribution and early wear on the bearing could result [12]. Heesterbeek et al. [14] indicated that excessive medial release may 
cause less external rotation of the femoral component. However, they used a gap tensioning device and goniometer, which may be less precise than the navigation system we used, and their study included only 32 knees with medial releases. Although Christensen et al. [6] showed more medial release results with less external rotation of the femoral component, no detailed gap data corresponding to each release step were provided in their report, and in their study, soft tissue release was the only variable considered to affect the femoral component rotation. Dennis et al. [11] noted that excessive varus tibial resection greater than $3^{\circ}$ is one factor associated with an internally implanted femoral component in TKA with the gap balancing technique. In the current study, the coronal angle (varus-valgus) of the resected tibia did not seem to be associated with internal rotation of the femoral component. The mean coronal angle of the resected tibia was almost neutral, and there were no outliers exceeding $\pm 3^{\circ}$ $[13,26,29,41]$. This may be because of the small variability in the coronal angle of the resected tibia. We suspect that excessive varus tibial resections are not common, with or without computer assistance [8, 31, 39, 42].

In the current study, navigation data regarding femoral component rotational alignment showed high accuracy compared with that of expected rotational alignment of the femoral component measured by pre- and postoperative CT scans. This finding is consistent with those of previous studies [13, 41]. However, those studies were based on the comparison between conventional and navigated TKAs. In those studies, although numerous outliers were consistently smaller in the navigated group than in the conventional group, it is difficult to conclude that intraoperative navigation data were accurate when compared with expected rotational profile measure with pre- and postoperative CT scans. Two recent studies compared the rotational profile of navigation data with that of postoperative $\mathrm{CT}$, which was similar to our method [7, 44]. However, Dahabreh et al. [7] reported that mismatch exceeding $3^{\circ}$ between postoperative $\mathrm{CT}$ and navigation measurements was $22 \%$, which was larger than ours (3.4\%). We suspect that difference between the two studies occurred because they used the measured resection-based navigation TKA (medial and lateral epicondylar referencing), which was different from ours (gap balancing-based technique, the most anterior and posterior points of the femur referencing). Dahabreh et al. [7] stated that landmark (medial and lateral epicondyles) registration errors are possible. This is indirect evidence of low reproducibility and reliability of bony landmarks for the measured resection, which others have commented on $[9,11,20,22]$. In addition, the number of cases in the study of Zambianchi et al. [44] was relatively small.

After considering preoperative coronal and axial alignment, PCL resection, coronal angle of the resected tibia, and extent of the medial release as possible associated factors, the extent of the medial release was the only factor we found that was associated with internal rotation of the femoral component in a gap balancing TKA. To avoid internal rotation of the femoral component, we recommend a carefully subdivided medial-releasing technique, especially for the superficial MCL, because once the superficial MCL has been completely released it cannot easily be restored. Further studies on safe and reproducible medial release techniques are needed.

\section{References}

1. Altman DG. Practical Statistics for Medical Research. 1st ed. London, UK: Chapman \& Hall; 1991.

2. Bellemans J. Multiple needle puncturing: balancing the varus knee. Orthopedics. 2011;34:e510-512.

3. Beltran J, Matityahu A, Hwang K, Jbara M, Maimon R, Padron M, Mota J, Beltran L, Sundaram M. The distal semimembranosus complex: normal MR anatomy, variants, biomechanics and pathology. Skeletal Radiol. 2003;32:435-445.

4. Berend ME, Davis PJ, Ritter MA, Keating M, Faris P, Meding JB, Malinzak RA. "Thicker" polyethylene bearings are associated with higher failure rates in primary total knee arthroplasty. $J$ Arthroplasty. 2010;25(6 suppl):17-20.

5. Chen W, Nagamine R, Kondo K, Todo M. Effect of medial softtissue releases during posterior-stabilised total knee arthroplasty. J Orthop Surg (Hong Kong). 2011;19:230-233.

6. Christensen CP, Stewart AH, Jacobs CA. Soft tissue releases affect the femoral component rotation necessary to create a balanced flexion gap during total knee arthroplasty. $J$ Arthroplasty. 2013;28:1528-1532.

7. Dahabreh Z, Scholes CJ, Giuffre B, Coolican MRJ, Parker DA. Lack of agreement between computer navigation and post-operative 2-dimensional computed tomography (CT) measurements for component and limb alignment in total knee arthroplasty (TKA). Knee. 2016;23:137-143.

8. Daines BK, Dennis DA. Gap balancing vs. measured resection technique in total knee arthroplasty. Clin Orthop Surg. 2014;6:18.

9. Davis ET, Pagkalos J, Gallie PA, Macgroarty K, Waddell JP, Schemitsch EH. Defining the errors in the registration process during imageless computer navigation in total knee arthroplasty: a cadaveric study. J Arthroplasty. 2014;29:698-701.

10. Dennis DA. Measured resection: an outdated technique in total knee arthroplasty. Orthopedics. 2008;31:940, 943-944.

11. Dennis DA, Komistek RD, Kim RH, Sharma A. Gap balancing versus measured resection technique for total knee arthroplasty. Clin Orthop Relat Res. 2010;468:102-107.

12. Fehring TK. Rotational malalignment of the femoral component in total knee arthroplasty. Clin Orthop Relat Res. 2000;380:72-79.

13. Harvie P, Sloan K, Beaver RJ. Computer navigation vs conventional total knee arthroplasty: five-year functional results of a prospective randomized trial. J Arthroplasty. 2012;27:667672.e1.

14. Heesterbeek PJ, Jacobs WC, Wymenga AB. Effects of the balanced gap technique on femoral component rotation in TKA. Clin Orthop Relat Res. 2009;467:1015-1022.

15. Hocking RR. Methods and Applications of Linear Models: Regression and the Analysis of Variance. 2nd ed. Hoboken, NJ: Wiley-Interscience; 2003. 
16. Hungerford DS. Measured resection: a valuable tool in TKA. Orthopedics. 2008;31:941-942.

17. Hunt NC, Ghosh KM, Athwal KK, Longstaff LM, Amis AA, Deehan DJ. Lack of evidence to support present medial release methods in total knee arthroplasty. Knee Surg Sports Traumatol Arthrosc. 2014;22:3100-3112.

18. Ishii Y, Noguchi H, Sato J, Todoroki K, Toyabe S. Retention of the posterior cruciate ligament does not affect femoral rotational alignment in TKA using a gap-balance technique. Knee Surg Sports Traumatol Arthrosc. 2014;22:3121-3126.

19. Ji HM, Jin DS, Han J, Choo HS, Won YY. Comparison of alternate references for femoral rotation in female patients undergoing total knee arthroplasty. Knee Surg Sports Traumatol Arthrosc. 2016;24:2402-2406.

20. Katz MA, Beck TD, Silber JS, Seldes RM, Lotke PA. Determining femoral rotational alignment in total knee arthroplasty: reliability of techniques. $J$ Arthroplasty. 2001;16:301-305.

21. Kim MW, Koh IJ, Kim JH, Jung JJ, In Y. Efficacy and safety of a novel three-step medial release technique in varus total knee arthroplasty. J Arthroplasty. 2015;30:1542-1547.

22. Kinzel V, Ledger M, Shakespeare D. Can the epicondylar axis be defined accurately in total knee arthroplasty? Knee. 2005;12:293296.

23. LaPrade RF, Engebretsen AH, Ly TV, Johansen S, Wentorf FA, Engebretsen L. The anatomy of the medial part of the knee. $J$ Bone Joint Surg Am. 2007;89:2000-2010.

24. LaPrade RF, Morgan PM, Wentorf FA, Johansen S, Engebretsen L. The anatomy of the posterior aspect of the knee: an anatomic study. J Bone Joint Surg Am. 2007;89:758-764.

25. Lee DS, Song EK, Seon JK, Park SJ. Effect of balanced gap total knee arthroplasty on intraoperative laxities and femoral component rotation. J Arthroplasty. 2011;26:699-704.

26. Lutzner J, Krummenauer F, Wolf C, Gunther KP, Kirschner S. Computer-assisted and conventional total knee replacement: a comparative, prospective, randomised study with radiological and CT evaluation. J Bone Joint Surg Br. 2008;90:1039-1044.

27. Matsueda M, Gengerke TR, Murphy M, Lew WD, Gustilo RB. Soft tissue release in total knee arthroplasty: cadaver study using knees without deformities. Clin Orthop Relat Res. 1999;366:264273.

28. Matsumoto T, Kubo S, Muratsu H, Matsushita T, Ishida K, Kawakami Y, Oka S, Matsuzaki T, Kuroda Y, Nishida K, Akisue T, Kuroda R, Kurosaka M. Different pattern in gap balancing between the cruciate-retaining and posterior-stabilized total knee arthroplasty. Knee Surg Sports Traumatol Arthrosc. 2013;21:2338-2345.

29. Matziolis G, Krocker D, Weiss U, Tohtz S, Perka C. A prospective, randomized study of computer-assisted and conventional total knee arthroplasty: three-dimensional evaluation of implant alignment and rotation. $J$ Bone Joint Surg Am. 2007;89:236-243.
30. McPherson EJ. Patellar tracking in primary total knee arthroplasty. Instr Course Lect. 2006;55:439-448.

31. Mihalko WM, Saleh KJ, Krackow KA, Whiteside LA. Soft-tissue balancing during total knee arthroplasty in the varus knee. $J A m$ Acad Orthop Surg. 2009;17:766-774.

32. Mihalko WM, Whiteside LA, Krackow KA. Comparison of ligament-balancing techniques during total knee arthroplasty. $J$ Bone Joint Surg Am. 2003;85(suppl. 4):132-135.

33. Mullaji AB, Shetty GM. Surgical technique: computer-assisted sliding medial condylar osteotomy to achieve gap balance in varus knees during TKA. Clin Orthop Relat Res. 2013;471:14841491.

34. Okazaki K, Miura H, Matsuda S, Takeuchi N, Mawatari T, Hashizume M, Iwamoto Y. Asymmetry of mediolateral laxity of the normal knee. J Orthop Sci. 2006;11:264-266.

35. Peduzzi P, Concato J, Kemper E, Holford TR, Feinstein AR. A simulation study of the number of events per variable in logistic regression analysis. J Clin Epidemiol. 1996;49:1373-1379.

36. Rhoads DD, Noble PC, Reuben JD, Mahoney OM, Tullos HS. The effect of femoral component position on patellar tracking after total knee arthroplasty. Clin Orthop Relat Res. 1990;260:43-51.

37. Rhoads DD, Noble PC, Reuben JD, Tullos HS. The effect of femoral component position on the kinematics of total knee arthroplasty. Clin Orthop Relat Res. 1993;286:122-129.

38. Romero J, Stahelin T, Binkert C, Pfirrmann C, Hodler J, Kessler $\mathrm{O}$. The clinical consequences of flexion gap asymmetry in total knee arthroplasty. J Arthroplasty. 2007;22:235-240.

39. Scott WN, ed. Insall \& Scott Surgery of the Knee. 4th ed. Philadelphia, PA: Elsevier Churchill Livingstone; 2006.

40. Seo JG, Moon YW, Jo BC, Kim YT, Park SH. Soft tissue balancing of varus arthritic knee in minimally invasive surgery total knee arthroplasty: comparison between posterior oblique ligament release and superficial MCL release. Knee Surg Relat Res. 2013;25:60-64.

41. Stockl B, Nogler M, Rosiek R, Fischer M, Krismer M, Kessler O. Navigation improves accuracy of rotational alignment in total knee arthroplasty. Clin Orthop Relat Res. 2004;426:180186.

42. Verdonk PC, Pernin J, Pinaroli A, Ait Si Selmi T, Neyret P. Soft tissue balancing in varus total knee arthroplasty: an algorithmic approach. Knee Surg Sports Traumatol Arthrosc. 2009;17:660666.

43. Yagishita K, Muneta T, Ikeda H. Step-by-step measurements of soft tissue balancing during total knee arthroplasty for patients with varus knees. J Arthroplasty. 2003;18:313-32.

44. Zambianchi F, Luyckx T, Victor J, Digennaro V, Giorgini A, Catani F. How toe improve femoral component rotational alignment in computer-assisted TKA. Knee Surg Sports Traumatol Arthrosc. 2014;22:1805-1811. 